\title{
Surface properties modulate protein corona formation and determine cellular uptake and cytotoxicity of silver nanoparticles
}

Marianna Barbalinardo, ${ }^{\mathrm{a}}$ Jessika Bertacchini, ${ }^{\mathrm{b}}$ Linda Bergamini, ${ }^{\mathrm{c}}$ Maria Sara Magarò, ${ }^{\mathrm{b}}$ Luca Ortolani, ${ }^{\mathrm{d}}$ Alessandra Sanson, ${ }^{\mathrm{c}}$ Carla Palumbo, ${ }^{\mathrm{b}}$ Massimiliano Cavallini ${ }^{\mathrm{a}}$ and Denis Gentili*a

a) Consiglio Nazionale delle Ricerche, Istituto per lo Studio dei Materiali Nanostrutturati (CNRISMN), via P. Gobetti 101, 40129 Bologna (Italy)

b) Department of Biomedical, Metabolic and Neural Sciences, Section of Human Morphology, University of Modena and Reggio Emilia, Via del Pozzo 71, 41124 Modena (Italy)

c) Consiglio Nazionale delle Ricerche, Istituto di Scienza e Tecnologia dei Materiali Ceramici (CNRISTEC), via Granarolo 64, 48018 Faenza (Italy)

d) Consiglio Nazionale delle Ricerche, Istituto per la Microelettronica e Microsistemi (CNR-IMM), via P. Gobetti 101, 40129 Bologna (Italy)

Keywords: Nanoparticles, Surface properties, Protein corona, Cellular uptake, Cytotoxicity.

Abstract: Nanoparticles (NPs) have been studied for biomedical applications, ranging from prevention, diagnosis and treatment of diseases. However, the lack of the basic understanding of how NPs interact with the biological environment has severely limited their delivery efficiency to target tissue and clinical translation. Here, we show the effective regulation of the surface properties of NPs, by controlling the surface ligand density, and their effect in serum protein adsorption, cellular uptake and cytotoxicity. Surface properties of NPs are tuned through the controlled replacement of native ligands, which favor protein adsorption, with ligands able to increase protein adsorption resistance. The extent and composition of the protein layer adsorbed on NPs are strongly correlated to the degree of ligands replaced on their surface and, while BSA is the most abundant protein detected, ApoE is the one whose amount is most affected by surface properties. Increasing the protein resistance, cellular uptake and cytotoxicity in mouse embryonic fibroblasts of NPs are drastically reduced, but surface coating has not effects on the process by which NPs mainly induce cell death. Overall, this study reveals that the tuning of surface properties of NPs allows us to regulate their biological outcomes by controlling their ability to adsorb serum proteins. 


\section{Introduction}

Over the past decades, nanoparticles (NPs) have been extensively studied for biomedical applications, ranging from prevention, early diagnosis and treatment of diseases as their unique and highly tunable physical, chemical, and biological properties promise to overcome some critical shortcomings of conventional medicine, such as poor bioavailability and low target specificity, as well as facing the challenges related to improving the quality of life of patients during treatments. However, the lack of the basic understanding of how NPs interact with the biological environment and, in turn, with living systems has severely limited their potential in medicine.[1-6] Despite the significant progress has been made to overcome some critical limits, to date, independently of material (organic or inorganic) and targeting strategy (active or passive), the delivery of NPs to target tissue is less than $1 \%$ of the injected dose and their clinical translation has been limited. Although NPs can be engineered with precise functional properties for specific and nonspecific cellular targeting, they have to face the physical and biological barriers before reaching target tissue, resulting in a very low nanoparticle delivery efficiency.[7,8] There is no simple correlation between biological responses and synthetic identity of NPs (i.e., size, shape and surface properties postsynthesis) as, in biological fluids, their physicochemical properties can drastically change following the interactions and adsorption onto their surface of extracellular proteins and other biological molecules, creating the so-called protein corona. The structure and composition of the protein corona, which depend on the combination of synthetic identity of NPs with nature of the biological fluid and exposure time, can be sufficiently long lived to determine the biological identity of NPs and, therefore, can critically regulate their physiological responses.[5,9-11] Thus, elucidating the role of each physicochemical parameter of NPs is needed to control their identity in biological media and, in turn, develop effective strategies to design long-circulating and target-specific NPs with increased delivery efficiency and improved therapeutic response.[12-16] Among all physicochemical parameters that affect the nature and amount of proteins adsorption, the surface properties of NPs have been reported to play the most significant role on the formation of the protein corona.[17,18] We have recently demonstrated that surface coating of silver nanoparticles (AgNPs) strongly affects the adsorption of serum proteins and, in turn, their cellular uptake and cytotoxicity in mouse embryonic fibroblasts. In particular, citratecoated AgNPs are promptly coated and stabilized by a protein corona and show a time- and dosedependent toxicity, while the passivation of the NP surface with an oligo(ethylene glycol) (OEG)terminated alkanethiol drastically reduces both the adsorption of proteins and the cytotoxicity.[19,20] 


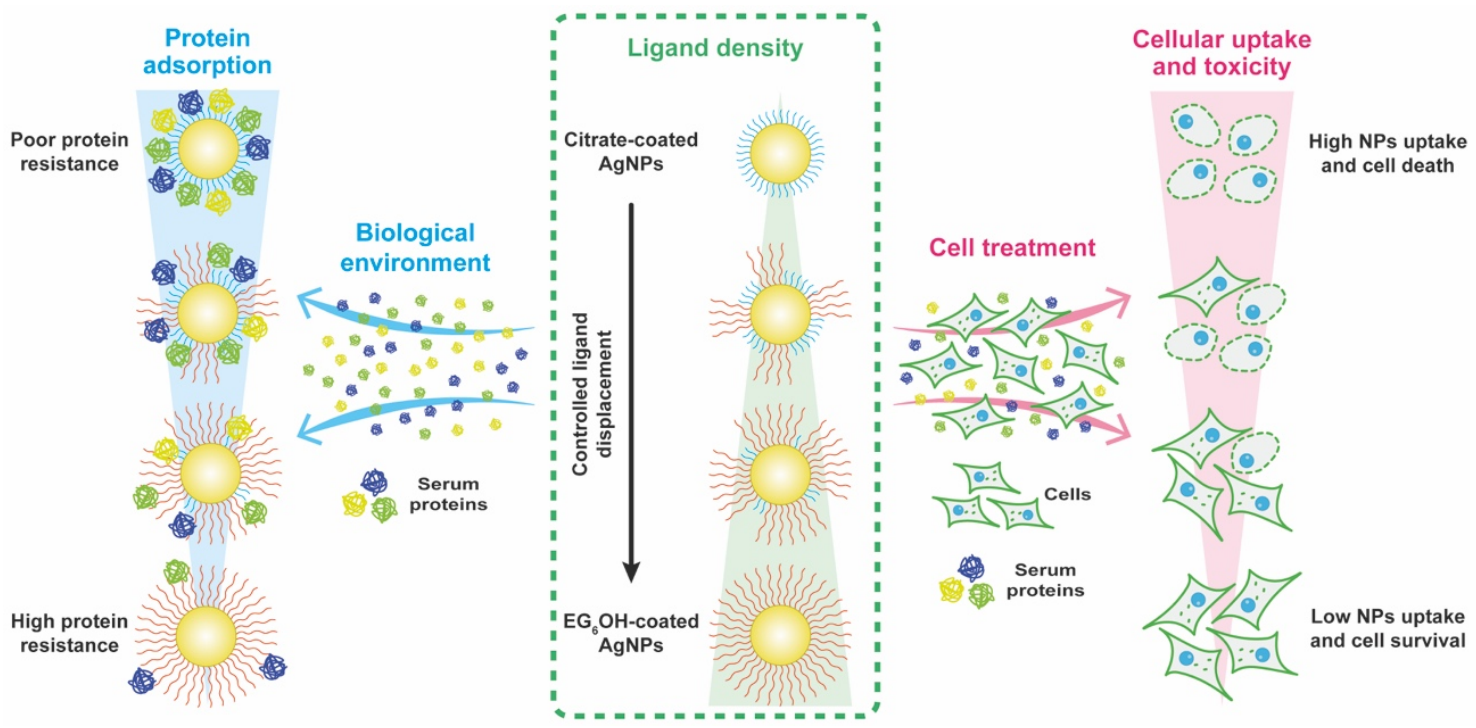

Scheme 1 Schematic illustration of the effect of ligand $\left(\mathrm{EG}_{6} \mathrm{OH}\right)$ density on protein adsorption (left), cellular uptake and cytotoxicity (right) of AgNPs.

In the present work, we report the role of surface coverage of nanoparticles in serum protein adsorption, cellular uptake and cytotoxicity. As schematically shown in Scheme 1, we use citratecoated AgNPs and gradually modify their surface properties through the controlled displacement of citrate ions with an OEG-terminated alkanethiol, namely (11-mercaptoundecyl)hexa(ethylene glycol)

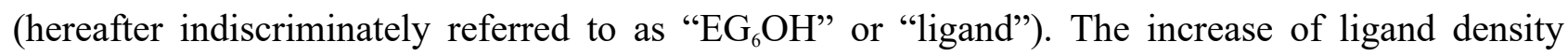
gradually increases the resistance to protein adsorption of the AgNPs, thus drastically affecting both composition and amount of proteins that compose their protein corona. These changes in the protein corona have, in turn, a significant impact on the biological identity of nanoparticles and, therefore, on their interactions with cells. We show that the uptake and cytotoxic activity of AgNPs on mouse embryonic fibroblasts strongly correlates with the surface properties, in fact both proportionally decrease with increasing the ligand density, but the process by which AgNPs mainly induce cell death remains the same. These results reveal that the surface properties of NPs regulate their interaction with cells and, in turn, dictate their biological outcomes. The synergism between the surface coverage and biological identity of NPs can be exploited for their rational design and engineering, thus thoroughly tailoring their interactions with living systems to specific applications and minimizing the side effects. 


\section{Results and discussion}

Alkanethiols terminated with an OEG unit have been extensively studied for their ability to form protein resistant self-assembled monolayers (SAMs) on gold and silver surfaces,[21-24] even when used in mixed SAMs.[25-27] The resistance to protein adsorption is determined by the internal and terminal hydrophilicity, the number of ethylene glycol units and lateral packing density of OEGterminated alkanethiol used. The lateral packing density, which in turn determines the molecular conformation of the OEG moieties, can be affected both by the assembly solvent and the substrate material.[22,23] Among all, $\mathrm{EG}_{6} \mathrm{OH}$ is an OEG-terminated alkanethiol able to form robust protein resistant SAMs on planar silver surfaces,[21,22] as well as on AgNPs,[19,20] regardless of the assembly solvent used.[23] Therefore, we have tuned the protein resistance properties of AgNPs through the controlled replacement of citrate ions, which favor surface protein adsorption,[19] with $\mathrm{EG}_{6} \mathrm{OH}$. Citrate-coated AgNPs having an average diameter of $22 \pm 4 \mathrm{~nm}$, as determined from transmission electron microscopy (TEM) analysis (see SI - Fig. S1a, b), were prepared as previously reported[28] and functionalized with increasing density of $\mathrm{EG}_{6} \mathrm{OH}$. Because thiol containing molecules bind strongly to the gold and silver surfaces and can displace other weakly bound ligands, such as citrate ions,[29] the density of the $\mathrm{EG}_{6} \mathrm{OH}$ on the nanoparticles surface was controlled by varying the $\mathrm{EG}_{6} \mathrm{OH}$-to-nanoparticle ratio and quantified by a thiol depletion assay (see Experimental section).
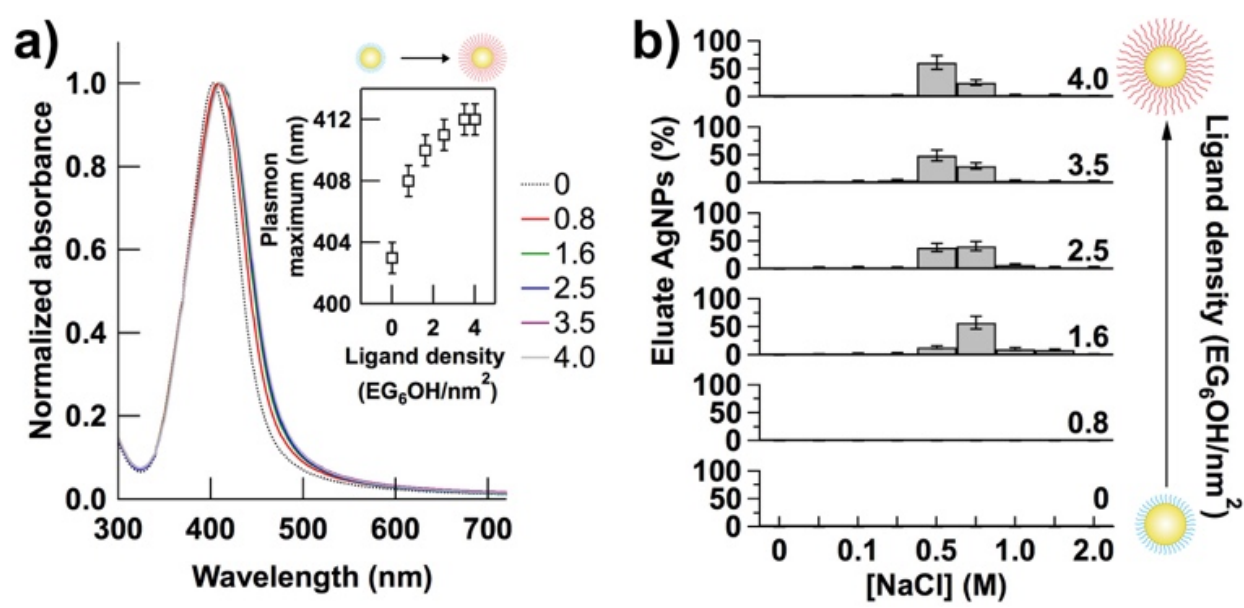

Fig. 1 (a) UV-Vis absorption spectra of AgNPs in sodium citrate $(2 \mathrm{mM})$ as a function of $\mathrm{EG}_{6} \mathrm{OH}$ density. Inset: Dependence of the plasmon band maximum on the $\mathrm{EG}_{6} \mathrm{OH}$ density. (b) Elution profile of $\mathrm{AgNPs}$ as a function of $\mathrm{EG}_{6} \mathrm{OH}$ density on anion-exchange chromatography using increasing concentrations of $\mathrm{NaCl}$. 
The studied ligand densities range from AgNPs coated exclusively with citrate ions, i.e. NPs with $\mathrm{EG}_{6} \mathrm{OH}$ density $=0$ (hereafter indiscriminately referred to such NPs as "0" or "citrate-coated AgNPs"), to saturating density of $\mathrm{EG}_{6} \mathrm{OH}\left(4.0 \pm 0.1 \mathrm{EG}_{6} \mathrm{OH} / \mathrm{nm}^{2}-\right.$ see SI). Noteworthy, the saturation ligand density is in good agreement with values reported on planar surfaces[21] and gold nanoparticles.[30,31] Citrate-coated AgNPs shows the characteristic localized surface plasmon resonance (LSPR) band centered at $400 \mathrm{~nm}(\mathrm{~S} 1 \mathrm{c}$ in SI) that is red-shifted, without shape variation, upon surface functionalization with the ligand (Fig. 1a and a zoom-in of it in SI - Fig S1d). We noticed the absence of broadening of the LSPR band also for very low density (i.e. 0.6-0.8 $\mathrm{EG}_{6} \mathrm{OH} / \mathrm{nm}^{2}$ ) thus revealing that there is no aggregation of AgNPs, contrarily to what we previously reported for gold nanoparticles.[32] Fig 1 inset shows the ligand density dependence of the plasmon band peak. Below the saturation density, the plasmon band maximum is found to red shift rapidly increasing the $\mathrm{EG}_{6} \mathrm{OH}$ density, as a result of the gradual change of refractive index of the surrounding environment.[33] Approaching saturation, the plasmon band maximum of AgNPs increases more slowly up to reach its maximum value. Noteworthy, this trend is reproducible on all batches of citratecoated AgNPs we have prepared and functionalized with $\mathrm{EG}_{6} \mathrm{OH}$ (see for a different batch SI - Fig. $\mathrm{S} 1 \mathrm{e})$. The gradual replacement of citrate ions adsorbed on the nanoparticle surface with $\mathrm{EG}_{6} \mathrm{OH}$ molecules was further confirmed by anion-exchange chromatography on diethylaminoethyl (DEAE) sepharose. Although AgNPs bind to the positively charged resin regardless of $\mathrm{EG}_{6} \mathrm{OH}$ density, because of they show a negative zeta potential (see below), their elution profile is drastically different as a function of surface coverage. As shown in Fig 1b, AgNPs with low densities $\left(\leq 0.8 \mathrm{EG}_{6} \mathrm{OH} / \mathrm{nm}^{2}\right)$ bind irreversibly to the DEAE resin as they were not eluted even with high concentration of $\mathrm{NaCl}$ (2M). However, increasing the ligand density, the elution of AgNPs takes place at lower concentrations of $\mathrm{NaCl}$ and almost complete elution of NPs was obtained with $0.5 \mathrm{M} \mathrm{NaCl}$ approaching the saturation density. The gradual increase of AgNPs stability against irreversible adsorption onto resin is due to the replacement on their surface of weakly associated citrate ions, which can be easily displaced by the functional groups of the resin, with $\mathrm{EG}_{6} \mathrm{OH}$ that, on the contrary, bind strongly to the nanoparticles surface. In addition, as we already reported for gold nanoparticles, the replacement of citrate ions with $\mathrm{EG}_{6} \mathrm{OH}$ molecules leads to a decrease of electrostatic repulsions and a simultaneous increase of steric ones between the nanoparticles proportional to the extent of surface ligand substitution.[32] In agreement, electrolyte-induced aggregation test showed that citrate-coated AgNPs are unstable already at a salt concentration of $0.05 \mathrm{M}$, but the stability of NPs in saline solution increases following the increase of $\mathrm{EG}_{6} \mathrm{OH}$ density up to show a good stability also in $1 \mathrm{M}$ saline solution (the highest value tested) when the density reaches the saturation value (see SI - Fig. S2). 

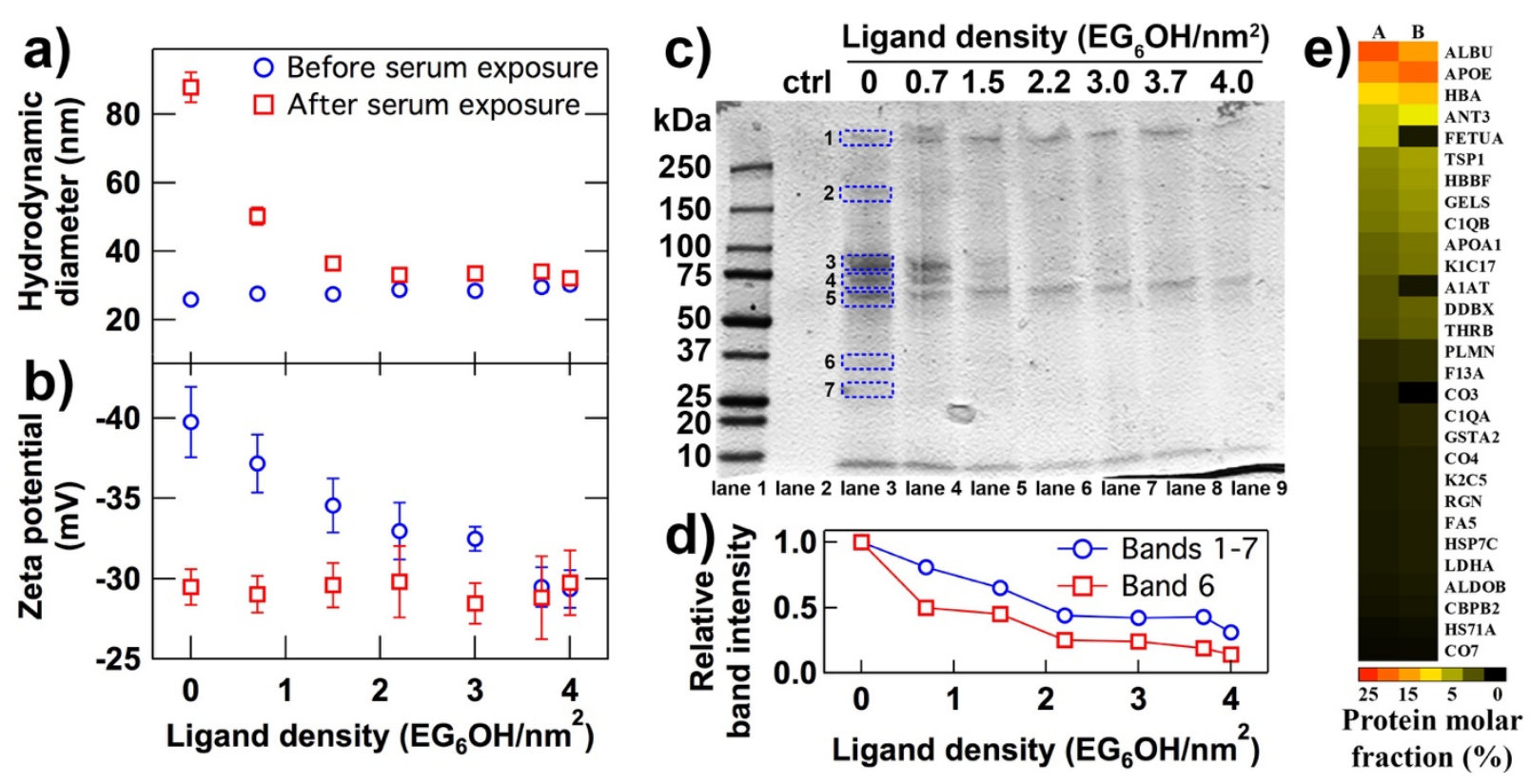

Fig. 2 (a) Hydrodynamic diameter and (b) zeta potential of AgNPs with different $\mathrm{EG}_{6} \mathrm{OH}$ density before (blue circle) and after (red square) incubation in growth media (exposure to serum proteins). (c) SDS-PAGE gel of adsorbed serum proteins on AgNPs with different $\mathrm{EG}_{6} \mathrm{OH}$ density. Molecular weight ladder is shown in lane 1. SDS-PAGE of products extracted from citrate-coated AgNPs not incubated in growth medium was included as control (ctrl, lane 2). (d) Densitometric analysis as a function of $\mathrm{EG}_{6} \mathrm{OH}$ density of PAGE scan showed in figure 2c. Relative band intensity was calculated by measuring for each lane identical areas corresponding to those marked by blue dashed squares only in lane 3 for convenience of clarity; (blue circle) sum of all (1-7) areas for each lane and (red square) only area 6. (e) Heat map of protein molar fractions identified and determined by proteomic mass spectrometry in the areas marked in lane 3 of figure $2 \mathrm{c}$ by blue dashed squares: sum of (A) 1-7 and (B) 2, 3, 4, 6 and 7 areas. Values were calculated from the emPAI of each protein identified by LC-MS/MS according to the equation: Protein content $(\mathrm{mol} \%)=e m P A I / \sum(e m P A I) .[34]$ Abbreviated protein names are explained in the SI - Table S1.

We have recently reported the key role of the adsorption of serum proteins onto surface of AgNPs in mediate their cellular uptake and cytotoxic activity.[19] Therefore, to investigate the effect of $\mathrm{EG}_{6} \mathrm{OH}$ density on serum protein adsorption, we measured the change of hydrodynamic diameter and zeta potential $(\zeta)$ of the NPs after incubation in growth medium containing fetal bovine serum (FBS) and subsequent extensive purification to remove unbound or weakly adsorbed proteins (see Experimental section). As we already reported, the mean hydrodynamic diameter of citrate-coated AgNPs is strongly increased by the incubation in serum-containing media. However, as shown in Fig. 2a, the discrepancy between the hydrodynamic diameter before and after incubation in cellular 
medium becomes less significant increasing the density of $\mathrm{EG}_{6} \mathrm{OH}$ and disappears, within the experimental error, when the saturation value is reached, revealing that the extent of protein adsorption is strictly correlated with surface coverage. The zeta potential shows a trend that is completely in agreement with what was observed with hydrodynamic diameters; the effect of the exposure to serum proteins on the $\zeta$ value of the AgNPs decreases following the increase of the $\mathrm{EG}_{6} \mathrm{OH}$ density and becomes insignificant when approaching the saturation density (Fig. 2b). To further investigate the role of surface coverage, after incubation in growth medium containing FBS and subsequent purification, serum proteins adsorbed on the surface of AgNPs were isolated and separated using polyacrylamide gel electrophoresis (PAGE) coupled with a protein stain (see Experimental section). As shown in Fig. 2c, proteins separated from citrate-coated AgNPs (lane 3) showed several bands, marked by blue dashed squares and labeled 1-7, that were not present in the control (ctrl, lane 2), confirming their ability to adsorb a broad range of serum proteins. The proteins isolated from AgNPs functionalized with higher ligand density showed similar band patterns (Fig. 2c, lanes 4-9). However, the intensity of some bands decreases with increasing ligand density up to disappear, revealing that the composition of the serum protein layer adsorbed on the AgNPs is strongly dependent on the surface coverage. In agreement, semiquantitative densitometry analysis of PAGE lanes shows that the total amount of adsorbed proteins contained in the areas corresponding to the bands 1-7 drastically decreases with increasing the $\mathrm{EG}_{6} \mathrm{OH}$ density (blue circle, Fig. 2d), however, the adsorption of proteins was not completely eliminated even at the saturation density. Noteworthy, our results are in agreement with what was observed for the adsorption of single protein on planar gold surface functionalized either with a mixed layer of $\mathrm{EG}_{6} \mathrm{OH}$ and n-alkanethiols[25,26] or with hydroxyl-terminated poly(ethylene glycol) (PEG)[35] and for the adsorption of serum proteins on gold nanoparticles functionalized with methoxy-terminated PEG.[36]

A more detailed investigation of the protein corona composition was performed using label-free liquid chromatography mass spectrometry (LC-MS/MS). Proteins separated in the seven bands from citrate-coated AgNPs (lane 3, Fig. 2c) were excised, digested with trypsin and analyzed by LCMS/MS. Protein molar fractions of serum proteins that compose the corona of citrate-coated AgNPs are reported in Fig. 2e (column A) and summarized in Table S1 in the SI. A total of 29 serum proteins were identified in seven bands and their relative abundances were estimated quantitatively by calculating their exponentially modified protein abundance index (emPAI).[34] Overall, the most abundant protein detected in the corona is bovine serum albumin (BSA, 21\% mol) followed by apolipoprotein E (ApoE, 16\% mol), which is a protein involved in lipids transport and uptake.[37,38] Both proteins were identified on different types of nanoparticles but, while the adsorption of albumin has been reported to protect nanomaterials from opsonization (dysopsonin effect), thus leading to an 
increase in their blood circulation times,[39-41] adsorption of ApoE is reported to mediate the interaction of nanomaterials with low-density lipoprotein receptors (LDLR), promoting their uptake into endothelial cells[37,38] and hepatocytes.[42] As mentioned above, the protein pattern changes with surface coverage, however, not all the bands (1-7 in Fig. 2c) show a significant decrease of intensity with increasing ligand density. As shown in Fig. 2c, bands 2, 3, 4, 6, and 7 show a marked decrease of the intensity upon increase of ligand density, while bands 1 and 5 are not affected significantly, suggesting that not all proteins composing the corona of citrate-coated AgNPs are affected by replacement of citrate ions with $\mathrm{EG}_{6} \mathrm{OH}$ molecules. Therefore, for a deeper insight, we compared the relative protein abundance calculated considering the sum of all (1-7) bands versus the relative protein abundance calculated considering only bands whose intensity decreases more drastically with the increase of ligand density (i.e. bands 2, 3, 4, 6, and 7 in Fig. 2c). As schematically reported in Fig. 2e and summarized in Table S2 in the SI, while BSA is the most abundant protein that composes the corona (Fig. 2e, column A), ApoE is the most abundant protein when only PAGE bands whose intensity is clearly affected by the surface coverage are considered (Fig. 2e, column B), revealing that content of ApoE in the corona is more affected by the ligand density than that of BSA.
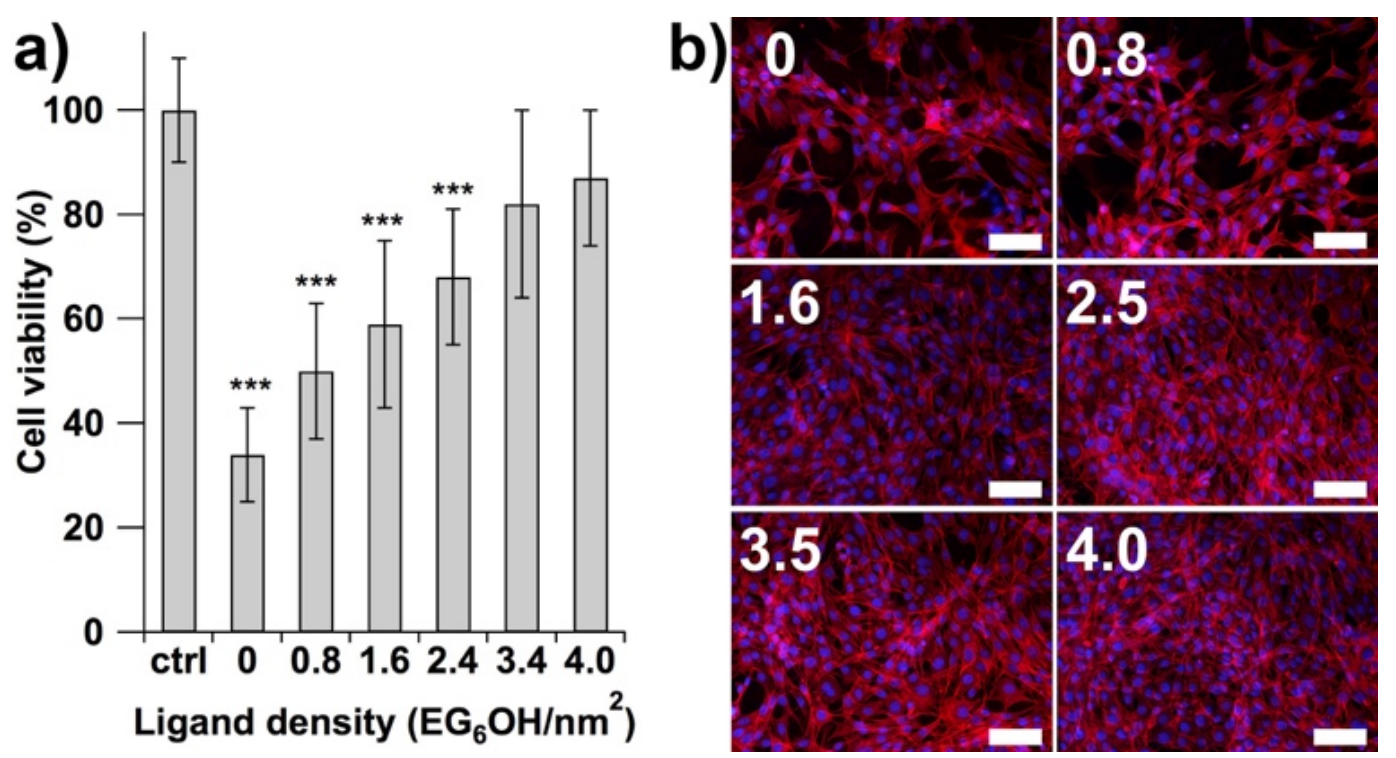

Fig. 3 (a) Cell viability of NIH-3T3 cells after $24 \mathrm{~h}$ of treatment with $40 \mu \mathrm{g} / \mathrm{mL}$ of AgNPs as a function ligand density. Data are presented as mean \pm standard deviation (SD). Statistical analysis was performed using ANOVA followed by Turkey's test. ***p $<0.001$ denotes significant differences respect to control (ctrl, cells treated with vehicle solution). (b) Fluorescence micrographs of cells labeled specifically for actin (red) and the nucleus (blue) after $24 \mathrm{~h}$ of incubation with 40 $\mu \mathrm{g} / \mathrm{mL}$ of AgNPs as a function ligand density $\left(\mathrm{EG}_{6} \mathrm{OH} / \mathrm{nm}^{2}\right)$ (Scale bar: $\left.100 \mu \mathrm{m}\right)$. 
In addition, since ApoE was identified only in band 6, we compared the trend of intensity of this band with the sum of all bands (1-7); as revealed by densitometric analysis, the amount of proteins in band 6 (red square, Fig. 2d) decreases more drastically with increasing $\mathrm{EG}_{6} \mathrm{OH}$ density than that of the sum of all bands (blue circle, Fig. 2d). Taking into account that ApoE represents almost the $60 \%$ mol of proteins contained in band 6 (see SI - Table S3), these results further confirm that adsorption of ApoE, more than those of $\mathrm{BSA}$, is influenced by the $\mathrm{EG}_{6} \mathrm{OH}$ density.

To evaluate the impact of $\mathrm{EG}_{6} \mathrm{OH}$ density on the interactions between the NPs and living systems, we performed studies of toxicity, based on 3-(4,5-dimethyl-2-thiazolyl)-2,5-diphenyltetrazolium bromide (MTT) assay, and cellular uptake using inductively coupled plasma optical emission spectroscopy (ICP-OES). Mouse embryonic fibroblasts (NIH-3T3) were selected as a model cell line and exposed to $40 \mu \mathrm{g} \mathrm{mL}-1$ of AgNPs, since we have recently showed their susceptibility to the coating-dependent toxicity of AgNPs.[19,20] After 24h of exposure to NPs, as shown in Fig. 3a, the MTT viability assay revealed that the surface coverage significantly determines the toxic activity of the AgNPs; cell viability drastically increases with increasing $\mathrm{EG}_{6} \mathrm{OH}$ density on the surface of AgNPs and, in agreement with what was previously observed,[19] becomes not significantly affected compared to control (ctrl), i.e. cells treated with vehicle solution ( $2 \mathrm{mM}$ sodium citrate), when the saturation density of the ligand on the NPs surface is reached. In agreement with viability results, double fluorescence labeling of actin (red) and nucleus (blue) showed surface coating-depend toxicity of AgNPs (Fig. 3b). At low $\mathrm{EG}_{6} \mathrm{OH}$ density values, cytoskeleton size and density of fibroblasts treated with AgNPs are reduced, thus revealing an impaired ability to replicate and less adhesion to the substrate. The increase of ligand density clearly decreases the toxic effects of AgNPs, in fact, approaching the saturation density, the morphology and density of cells treated with AgNPs is similar to that of cells treated with vehicle solution (control, see SI - Fig. S3).

For a deeper understanding of the role of surface coating on the mode of cell death, NIH-3T3 cells were double-stained with acridine orange/ethidium bromide (AO/EB) following $48 \mathrm{~h}$ of exposure to AgNPs. Data were extracted from the fluorescence microscopy images based on the percentages of green (viable cells), red (necrotic cells), yellow (early apoptosis), and orange (late apoptosis) fluorescence cell,[43] and results are shown in Fig. 4a. Consistently with the MTT viability assay, inhibition of cell proliferation after $48 \mathrm{~h}$ of exposure to AgNPs shows a trend that is completely in agreement with what was observed at $24 \mathrm{~h}$ (Fig. 3a), in fact the increase of $\mathrm{EG}_{6} \mathrm{OH}_{\text {density }}$ on the nanoparticle surface drastically reduces their cytotoxic activity, further confirming its key role on the interaction between NPs and cells. Moreover, double-staining assay clearly showed that, independently of the ligand density, AgNPs induce cell death leading mainly to their apoptosis and, 
to a lesser extent, necrosis. These results are in agreement with what was previously observed.[4447] Noteworthy, while the percentage of total apoptotic cells is inversely proportional to $\mathrm{EG}_{6} \mathrm{OH}$ density, there is no correlation with percentage of necrotic cells, however in all cases necrosis is the least relevant cell death process. From these results, we can infer that, although the surface coverage plays a key role on the extent of cytotoxic activity, the process by which AgNPs mainly induce cell death remains the same independently of the surface coverage of the nanoparticles. Considering that AgNPs carry out their toxic effects after cell internalization,[19,48] these results suggested that $\mathrm{EG}_{6} \mathrm{OH}$ density determines the toxic activity of $\mathrm{AgNPs}$ by regulating their cellular uptake. In order to probe whether variation of surface coverage was correlated with variation of NP internalization, we have assessed the relationship between ligand density and uptake of AgNPs in NIH-3T3 cells by ICP-OES analysis. The cellular uptake was measured following a $6 \mathrm{~h}$ incubation with the same concentration of AgNPs functionalized with different $\mathrm{EG}_{6} \mathrm{OH}$ density and extensive washing of NIH3 T3 cells, thereby silver per cell ratios represent AgNPs that were either tightly bound to the cell membrane or actually internalized (see Experimental section).
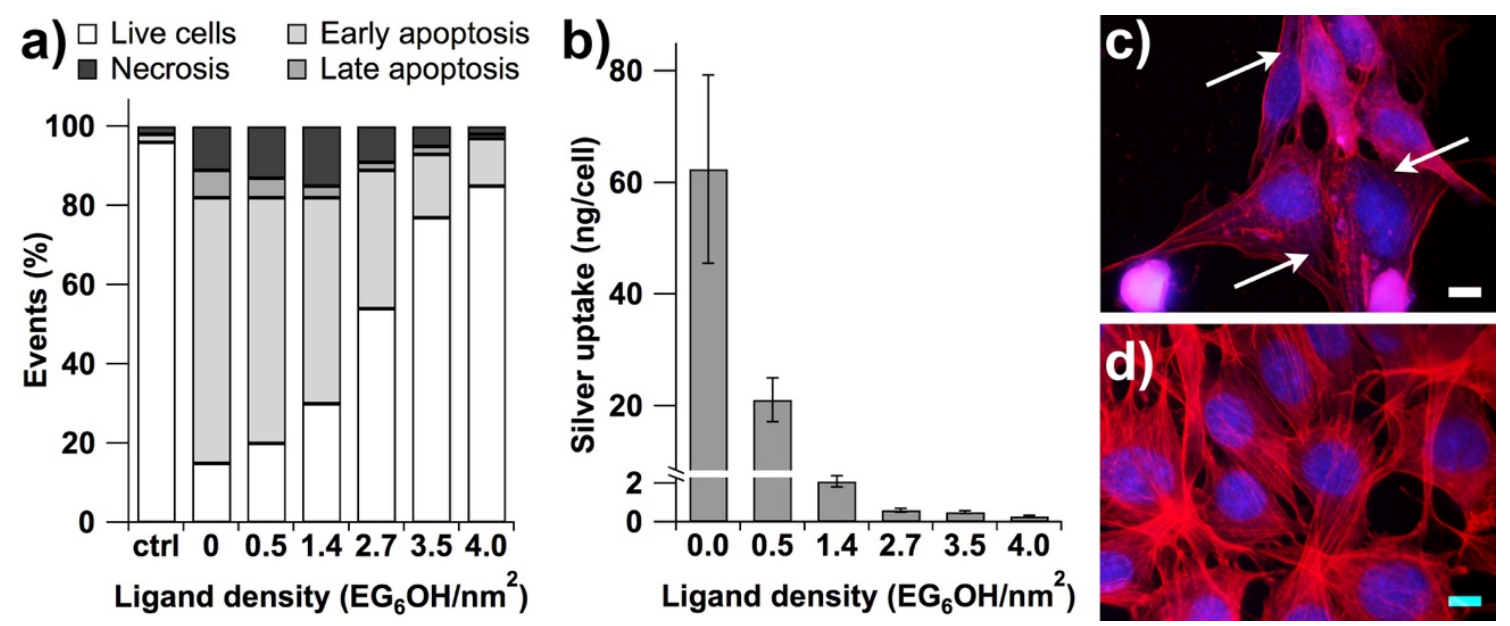

Fig. 4 (a) Double-staining assay of NIH-3T3 cells treated with $40 \mu \mathrm{g} / \mathrm{mL}$ of AgNPs after $48 \mathrm{~h}$ of incubation as a function ligand density. (b) Silver uptake of NIH-3T3 cells after $6 \mathrm{~h}$ of exposure to $40 \mu \mathrm{g} / \mathrm{mL}$ of AgNPs as a function ligand density. Data are presented as mean $\pm \mathrm{SD}$. Fluorescence micrographs of NIH-3T3 cells labeled specifically for actin (red) and the nucleus (blue) after $24 \mathrm{~h}$ of incubation with $40 \mu \mathrm{g} / \mathrm{mL}$ of AgNPs with ligand density (c) 0 and (d) $4.0 \mathrm{EG}_{6} \mathrm{OH} / \mathrm{nm}^{2}$, white arrows identify dark spots due to fluorescence quenching by metal NPs (Scale bar: $10 \mu \mathrm{m}$ ).

Note that short incubation time was chosen to ensure nanoparticles uptake, but preventing their toxic effects, in fact cell numbers after exposure to AgNPs was found to be similar to that of cells treated with vehicle solution ( $2 \mathrm{mM}$ sodium citrate). As shown in Fig. 4b, silver uptake drastically decreases 
with increasing the $\mathrm{EG}_{6} \mathrm{OH}$ density, in fact cells treated with citrate-coated AgNPs (ligand density = 0) show a silver uptake 200 times higher than those treated with nanoparticles completely coated with $\mathrm{EG}_{6} \mathrm{OH}$ (ligand density $=4$ ). Noteworthy, this large uptake difference was also qualitatively revealed by the presence of dark spots, due to fluorescence quenching by metal NPs, in the actin cytoskeleton image of cells treated with citrate-coated AgNPs (see white arrows in Fig. 4c), but they are not present in those of cells treated with AgNPs completely coated with $\mathrm{EG}_{6} \mathrm{OH}$ (Fig. 4d). These results clearly show that surface coating of AgNPs regulates their cellular uptake and, in turn, their cytotoxic effect.

\section{Conclusions}

In the present study, we have shown the role of surface coverage of nanoparticles in serum protein adsorption, cellular uptake and cytotoxicity. The surface properties of AgNPs were modulated through the controlled displacement of citrate ions with an oligo(ethylene glycol)-based ligand; the ligand density has been varied between no ligand (only citrate ions) and saturation. The exposure to serum-containing media revealed that extent and composition of the serum proteins layer adsorbed on the AgNPs, i.e. protein corona, is strongly dependent on the $\mathrm{EG}_{6} \mathrm{OH}$ density. The protein resistance of AgNPs increases following the increase of $\mathrm{EG}_{6} \mathrm{OH}$ density, however, the adsorption of proteins was not completely eliminated even at the saturation density. Although BSA was the most abundant protein detected in the corona, our results suggest that ApoE is the one whose amount is most affected by the ligand density. The different extent and composition of protein corona on the AgNPs significantly determine their cellular uptake and cytotoxicity, in fact both silver uptake and toxic activity drastically decrease with increasing the $\mathrm{EG}_{6} \mathrm{OH}$ density. However, surface coating has not effects on the process by which AgNPs mainly induce cell death that, in fact, occurs mainly through apoptosis regardless of ligand density. We can infer that the drastically different biological outcomes of AgNPs as a function of their surface coverage can be ascribed to the different ability to adsorb serum proteins which, in turn, regulate the toxic activity of AgNPs regulating their cellular uptake. In summary, our results reveal that the synergism between the surface coverage and biological identity of NPs can be exploited for their rational design and engineering, thus thoroughly tailoring the interaction of NPs with living systems to specific applications and minimizing the side effects.

\section{Experimental}

Materials. Silver nitrate $\left(\mathrm{AgNO}_{3}\right)$, sodium citrate $\left(\mathrm{C}_{6} \mathrm{H}_{5} \mathrm{O}_{7} \mathrm{Na}_{3}\right)$, tannic acid $\left(\mathrm{C}_{76} \mathrm{H}_{52} \mathrm{O}_{46}\right)$, (11mercaptoundecyl)hexa(ethylene glycol) $\left(\mathrm{EG}_{6} \mathrm{OH}\right)$, Ellman's reagent, dimethyl sulfoxide (DMSO), 3(4,5-dimethyl-2-thiazolyl)-2,5-diphenyltetrazolium bromide (MTT), MEM Non Essential Amino 
Acids (NEAA), Dulbecco's phosphate buffered saline (DPBS) and Dulbecco's modified Eagle medium (DMEM) were purchased from Sigma-Aldrich and used without further purification. All aqueous solutions were prepared with deionized water obtained using an ultrafiltration system (MilliQ, Millipore) with a measured resistivity above $18 \mathrm{M} \Omega$. Fetal bovine serum (FBS) was purchased from Gibco and heat-inactivated before use.

Synthesis of citrate-coated AgNPs. Citrate-stabilized AgNPs were prepared following the method reported elsewhere.[28,49] In brief, $100 \mathrm{~mL}$ of aqueous solution of sodium citrate $(5 \mathrm{mM})$ and tannic acid $(0.025 \mathrm{mM})$ was refluxed and an aqueous solution of silver nitrate $(1 \mathrm{~mL}, 25 \mathrm{mM})$ was added quickly. Then, the reaction mixture was refluxed for $30 \mathrm{~min}$, resulting in a bright yellow colloidal silver solution, and was then left to cool down to room temperature. The aqueous suspension of AgNPs was purified by two rounds of centrifugation (30000g for $2 \mathrm{~h}$ ) and resuspension in $10 \mathrm{~mL}$ of aqueous solution of sodium citrate $(2 \mathrm{mM})$.

Characterization of AgNPs. UV-Vis-NIR spectra were recorded on a Jasco V-560 UV-Vis spectrophotometer and Thermo Scientific Varioskan Flash Multimode Reader. Dynamic light scattering (DLS) and $\zeta$-potential measurement were performed in phosphate buffer $(1 \mathrm{mM}, \mathrm{pH}=7)$ and $\mathrm{KCl}(1 \mathrm{mM})$ on a NanoBrook Omni Particle Size Analyser (Brookhaven Instruments Corporation, USA) equipped with a $35 \mathrm{~mW}$ red diode laser (nominal $640 \mathrm{~nm}$ wavelength). AgNPs were characterized using Transmission Electron Microscopy (TEM) FEI Tecnai F20 ST, equipped with CCD Camera Gatan MSC704 and X-Rays EDS spectrometer EDAX EDS PV9761. TEM samples were prepared by drop casting 5 microliters of nanoparticle solutions onto a carbon-coated copper grid, wicked with filter paper to remove excess solution and dried in air for $20 \mathrm{~min}$. Average size and size distribution of citrate-stabilized AgNPs were measured using ImageJ software by counting 1000 particles. Silver concentrations were measured using Inductively Coupled Plasma Optical Emission Spectroscopy (ICP-OES) Varian Liberty 200. The concentration of silver was measured by ICP-OES titration at a $\lambda_{\mathrm{Ag}}=328.068$.

Saturation ligand density. The concentration of AgNPs was measured combining ICP-OES titration and TEM. The as-prepared water dispersed AgNPs were thoroughly mixed at room temperature overnight with a freshly prepared aqueous solution of $\mathrm{EG}_{6} \mathrm{OH}(\sim 2 \mathrm{mM})$ adjusting the added volume to achieve a stoichiometry of $\sim 10 \mathrm{EG}_{6} \mathrm{OH} / \mathrm{nm}^{2}$ that ensures to exceed the maximum ligand density. Nanoparticle-free control samples containing an equal concentration of $\mathrm{EG}_{6} \mathrm{OH}$ were prepared. After incubation, AgNPs were pelleted by centrifugation (13000g for $30 \mathrm{~min}$ ) and the supernatant containing the excess of thiol molecules was transferred to a new tube. The thiol concentrations both 
in the supernatant and in a nanoparticle-free control sample were measured with Ellman's reagent[50] and used to calculate the saturation ligand density.

Functionalization of AgNPs. The as-prepared water dispersed AgNPs were thoroughly mixed at room temperature overnight with a freshly prepared aqueous solutions of $\mathrm{EG}_{6} \mathrm{OH}$ adjusting the thiol concentration to achieve several samples with stoichiometry in the range between $0 \mathrm{EG}_{6} \mathrm{OH} / \mathrm{nm}^{2}$ and saturation. Nanoparticle-free control samples containing an equal concentration of $\mathrm{EG}_{6} \mathrm{OH}$ were prepared. After incubation, AgNPs were purified by four rounds of centrifugation (13000g for 30 min) and resuspension in aqueous solution of sodium citrate $(2 \mathrm{mM})$. The thiol concentrations of nanoparticle-free control samples were measured with Ellman's reagent[50] and used to calculate the ligand density.

Salt stability of AgNPs. AgNPs were mixed 1:19 volume ratio with aqueous solutions of $\mathrm{NaCl}$ in sodium citrate $(2 \mathrm{mM})$.

Ion Exchange Chromatography. Diethylaminoethyl (DEAE) Sepharose was prepared as specified by the manufacturer and equilibrated in sodium citrate $(2 \mathrm{mM}) .30 \mu \mathrm{L}$ of nanoparticles were incubated with $50 \mathrm{mg}$ of resin in a $1.5 \mathrm{~mL}$ tube for $1 \mathrm{~h}$ on a rotating wheel. The unbound nanoparticles were recovered and successive washes of the resin were then performed using phosphate buffer (10 $\mathrm{mM}$, $\mathrm{pH}=7$ ) with an increasing amount of $\mathrm{NaCl}$. The amount of recovered nanoparticles was quantified by the measurement of the absorbance at $400 \mathrm{~nm}$.

Serum Protein Adsorption. Silver nanoparticles $(100 \mu \mathrm{L})$ were mixed 1:5 volume ratio with complete medium (DMEM supplemented with 10\% (v/v) heat-inactivated FBS, 2 mM L-glutamine, $0.1 \mathrm{mM}$ MEM Non Essential Amino Acids (NEAA), $100 \mathrm{U} / \mathrm{mL}$ of penicillin and $100 \mathrm{U} / \mathrm{mL}$ of streptomycin) and incubated overnight at $37^{\circ} \mathrm{C}$. The nanoparticles were purified by three rounds of centrifugation $\left(22000 \mathrm{~g}\right.$ for $30 \mathrm{~min}$ at $4{ }^{\circ} \mathrm{C}$ ) and resuspension in $100 \mu \mathrm{L}$ of aqueous solution of phosphate buffer $(1 \mathrm{mM}, \mathrm{pH}=7)$ and $\mathrm{KCl}(1 \mathrm{mM})$.

Polyacrylamide Gel Electrophoresis (PAGE). AgNPs $(100 \mu \mathrm{L})$ were washed by two rounds of centrifugation $\left(13000 \mathrm{~g}\right.$ for $20 \mathrm{~min}$ at $4^{\circ} \mathrm{C}$ ) and resuspension in $40 \mu \mathrm{L}$ of Tris- $\mathrm{Cl}(10 \mathrm{mM}, \mathrm{pH} 7.4) .8$ $\mu 1$ of $6 \times$ Laemmli SDS sample reducing buffer (Bio Rad) was added to the nanoparticles and incubated at $100^{\circ} \mathrm{C}$ for $5 \mathrm{~min}$ to reduce and denature proteins on the surface of the particles. Nanoparticles were removed by centrifugation $\left(13000 \mathrm{~g}\right.$ for $20 \mathrm{~min}$ at $4^{\circ} \mathrm{C}$ ) and the supernatants containing the free proteins, along with molecular weight ladder (Bio Rad), were loaded on a 10\% SDS-PAGE and resolved at $100 \mathrm{~V}$ for $60 \mathrm{~min}$. Gel was fixed with a solution of $25 \%$ isopropyl alcohol, 
$10 \%$ glacial acetic acid for 60 min and stained with colloidal Coomassie Blue G-250 for $2 \mathrm{~h}$. Gel densitometry was performed using image $\mathrm{J}$ software.

Mass spectrometry analysis and protein identification. As reported above, protein separation was performed by PAGE using a $4-20 \%$ precast polyacrylamide gel (Bio Rad). The resulting gel was stained overnight with colloidal Coomassie Blue G-250 and seven bands, labeled A-G, were excised for Mass Spectrometry analysis. Protein digestion was performed following a protocol reported in literature.[51] Coomassie-stained bands were diced into $1 \mathrm{~mm}^{3}$ cubes, destained for 30 min with 50\% acetonitrile in $100 \mathrm{mM}$ ammonium bicarbonate buffer, dehydrated with $100 \%$ acetonitrile for $10 \mathrm{~min}$, reduced with $10 \mathrm{mM}$ DTT (1,4-Dithiothreitol, Sigma Aldrich) for $30 \mathrm{~min}$ at $56{ }^{\circ} \mathrm{C}$ and alkylated with $55 \mathrm{mM}$ iodoacetamide (IAA, Sigma Aldrich) for $30 \mathrm{~min}$ in the dark. After alkylation, gel pieces were dehydrated with 100\% acetonitrile and digested overnight with MS-grade trypsin (Thermo Fisher, $13 \mathrm{ng} / \mu \mathrm{l}$ in ammonium bicarbonate buffer $10 \mathrm{nM}$ with $10 \%$ acetonitrile) at $37^{\circ} \mathrm{C}$. The digestion products were extracted with a solution of $5 \%$ formic acid and acetonitrile $1: 2$ for $15 \mathrm{~min}$ at $37^{\circ} \mathrm{C}$ in a shaker and were then lyophilized using a SpeedVac Concentrator (Savant). Dry peptides from bands were resuspended in $40 \mu \mathrm{L}$ of a mixture of water: acetonitrile: formic acid 97:3:2, sonicated for 10 min at room temperature and centrifuged (12100g for $10 \mathrm{~min})$. Analyses were performed on an ESI Q Exactive Mass spectrometer (Thermo Scientific) controlled by Xcalibur (v. 29 build 2926) and interfaced with an Ultimate 3000 UHPLC pump. The column (Zorbax SB-C18 RRHT, 2.1x50mm, $1.8 \mu$ particle size, Agilent Technologies) was equilibrated with $0.3 \mathrm{ml} / \mathrm{min}$ of water $0.1 \%$ formic acid (A) with $2 \%$ acetonitrile (B); after sample injection (18 $\mu \mathrm{l}), \mathrm{B} \%$ was raised from 2 to $3 \%$, then linearly increased from 3 to $21 \%$ in $19 \mathrm{~min}$; B\% was then brought to $90 \%$ in 4 min and kept at $90 \%$ B for 3 min before the reconditioning step. The total runtime was $35 \mathrm{~min}$. ESI source was operated in positive mode; probe was heated at $290^{\circ} \mathrm{C}$, capillary temperature was set at $270{ }^{\circ} \mathrm{C}$; the following nitrogen flows (arbitrary units) were used to assist the ionization: Sheath Gas 40, Aux Gas 30, Sweep Gas 3; capillary voltage was set to $3.8 \mathrm{kV}$, S-Lens RF level was set at 45 (arbitrary units). Profile MS and $\mathrm{MS}^{2}$ spectra were recorded from 200 to $2000 \mathrm{~m} / \mathrm{z}$ in FULL MS/dd-MS² (TOP5) mode, at a resolution of 70000 and 17500, respectively. The five most intense multi-charged ions were selected for $\mathrm{MS}^{2}$ nitrogen-promoted collision-induced dissociation $(\mathrm{NCE}=28)$. A precursor active exclusion of 20 seconds was set; peptide-like isotope pattern ions were preferred. The mass spectrometer was calibrated before the start of the analyses. Raw data, converted into mascot generic format using MsConvert (v. 3.0.10730, ProteoWizard tools), were searched against Swiss-Prot (accessed Oct 2018; 6,002 sequences for Bos Taurus) for peptide sequences and C-RAP for contaminants with MASCOT (Version 2.4, Matrix Science, London, UK). Trypsin as proteolytic enzyme and carbamidomethyl cysteine as fixed modification were set in search parameters. Deamidated (NQ) and 
oxidated (M) were set as variable modifications. One missed cleavage was allowed. Mass tolerances were set at $10 \mathrm{ppm}$ for the precursor ions and $0.05 \mathrm{Da}$ for the product ions. Automatic decoy database search was used to estimate the false discovery rate, which was adjusted to $\leq 1 \%$.

Cell cultures. Mouse embryonic fibroblast (NIH-3T3) cells were cultured under standard conditions in the DMEM medium supplemented with $10 \%(\mathrm{v} / \mathrm{v})$ heat-inactivated FBS, $2 \mathrm{mM}$ L-glutamine, 0.1 mM MEM Non Essential Amino Acids (NEAA), $100 \mathrm{U} / \mathrm{mL}$ of penicillin and $100 \mathrm{U} / \mathrm{mL}$ of streptomycin in a humidified incubator set at $37^{\circ} \mathrm{C}$ with $5 \% \mathrm{CO}_{2}$. Cells were seeded in 96-well plates at a density $1 \times 10^{4}$ cells/well and grown for $24 \mathrm{~h}$ before exposure to nanoparticles.[52] For the control, cell culture medium was diluted with sodium citrate $(2 \mathrm{mM})$ to ensure that dilution of the medium by the solution of nanoparticles has no impact on the cell performance.

Actin and nucleus staining. Cells were fixed with 4\% paraformaldehyde in DPBS, washed with DPBS. They were then permeabilized with $0.001 \%$ Triton-X 100 . The cells were labelled with TRITC-conjugated phalloidin (FAK100, Merck Millipore) for $1 \mathrm{~h}$, followed by rinses with DPBS. Actin staining was critical to map local orientation of actin filaments within cells. Nuclear counterstaining was performed by incubation with DAPI (FAK100, Merck Millipore) for $3 \mathrm{~min}$, followed by rinses with DPBS. Samples were examined using a Nikon Eclipse 80i microscope equipped for fluorescence analysis.

Cells viability (Cytotoxicity assay). Cell viability was determined by 3-(4,5- di-methyl-2-thiazolyl)2,5-diphenyltetrazolium bromide (MTT) assay, measuring intracellular reduction of tetrazolium salts into purple formazan by viable cells.[53] Briefly, cells were seeded in 96-well plates with complete medium with or without nanoparticles for $24 \mathrm{~h}$ and $48 \mathrm{~h}$ in standard condition. After incubation times, the medium with or without AgNPs was discarded, and cells were washed with $100 \mu \mathrm{L}$ of DPBS and then $100 \mu \mathrm{L}$ of complete medium was added. Afterwards, $10 \mu \mathrm{L}$ of sterile MTT solution $(5 \mathrm{mg} / \mathrm{mL}$ in DPBS) was added to each well and incubated for $2 \mathrm{~h}$ at $37{ }^{\circ} \mathrm{C}$ with $5 \% \mathrm{CO}_{2}$. Subsequently, the medium was discarded and $200 \mu \mathrm{L}$ of DMSO was added to each sample to solubilize formazan crystals. Optical density (OD) was read on a microplate reader (Thermo Scientific Varioskan Flash Multimode Reader) at $550 \mathrm{~nm}$ as a working wavelength and $640 \mathrm{~nm}$ as a reference. Cell viability was calculated as the proportion of the mean OD of replicated wells relative to that of the control.

Fluorescence Microscopic Analysis of Cell Death. A combination of fluorescent DNA-binding dyes acridine orange (AO) and ethidium bromide (EB) were used to detect the cells death morphology, according to standard procedure.[54] Cells were seeded in 24-well plates at a density 4 $\times 10^{4}$ cells/well and grown for $24 \mathrm{~h}$ before exposure to nanoparticles. After $48 \mathrm{~h}$ of exposure, cells 
were treated with trypsine-EDTA 1x, neutralized with DPBS and collected by centrifugation. Then, fluorescent dyes AO (1 g/L) and EB (1 g/L) were added to the cellular pellet. Cell suspension was dropped on a glass slide and covered by a cover slip. Slides were observed with a Nikon eclipse $80 \mathrm{i}$ fluorescence microscope equipped with a UV-mercury lamp. Percentages of viable, apoptotic and necrotic cells were measured by analyzing 100 cells.

In vitro uptake of AgNPs. The uptake of AgNPs by NIH-3T3 cells was quantified using Inductively Coupled Plasma Optical Emission Spectroscopy (ICP-OES). AgNPs were incubated for 6h with preseeded NIH-3T3 cells in 12-well plates at a density 1 x $10^{5}$ cells/well. After exposure, the medium was discarded and cells were washed three times with DPBS to remove residual NPs. Then, cells were treated with trypsine-EDTA 1x, neutralized with DPBS, counted and collected by centrifugation. The recovered pellets were treated with $200 \mu \mathrm{L}$ of lysis buffer (RIPA buffer), digested overnight with freshly prepared aqua regia $\left(\mathrm{HNO}_{3}: \mathrm{HCl}=1: 3\right)$ and then diluted with $2 \% \mathrm{HNO}_{3}$. The concentration of silver was measured by ICP-OES at $328.068 \mathrm{~nm}$. Operating conditions of the ICPOES are listed below: RF power $1200 \mathrm{~W}$; plasma Ar flow rate of $12 \mathrm{~L} / \mathrm{min}$; nebulizer Ar flow rate: $0.70 \mathrm{~L} / \mathrm{min}$; uptake time: $25 \mathrm{~s}$; stabilization time: $15 \mathrm{~s}$. A series of silver standard solutions $(20,10$, $5,2.5,1.5,0.5,0 \mathrm{ppm})$ in $\mathrm{HNO}_{3} 2 \%$ were prepared to obtain a calibration curve used to determine the silver amount taken up by the cells in each sample, the concentration reported for each sample is the mean value of 5 different measures.

Statistical analysis. All data represented the mean \pm standard deviation (SD) of at least 4 independent culture experiments. Statistical significance was determined using a one-way analysis of variance with Turkey's test for multiple comparisons using Origin 8 software (OriginLab corporation). Differences were considered significant when $\mathrm{p}<0.005$ and $\mathrm{p}<0.001$.

\section{Corresponding Author}

Denis Gentili - Consiglio Nazionale delle Ricerche, Istituto per lo Studio dei Materiali Nanostrutturati (CNR-ISMN), via P. Gobetti 101, 40129 Bologna (Italy); orcid.org/0000-0002-75992804; Email: denis.gentili@cnr.it

\section{Conflicts of interest}

There are no conflicts to declare. 


\section{Acknowledgements}

The authors acknowledge the Fondazione Cassa di Risparmio di Modena for funding the UHPLCESI-Q Exactive system at the Centro Interdipartimentale Grandi Strumenti (CIGS). M.B. was supported by the project MIUR-PRIN prot. 2017YH9MRK

\section{References}

[1] Chen, G. Y., Roy, I., Yang, C. H. \& Prasad, P. N. Nanochemistry and Nanomedicine for Nanoparticle-based Diagnostics and Therapy. Chem. Rev. 116, 2826-2885, (2016).

[2] Cheng, L., Wang, C., Feng, L., Yang, K. \& Liu, Z. Functional Nanomaterials for Phototherapies of Cancer. Chem. Rev. 114, 10869-10939, (2014).

[3] Barreto, J. A. et al. Nanomaterials: Applications in Cancer Imaging and Therapy. Adv. Mater. 23, H18-H40, (2011).

[4] Lim, E.-K. et al. Nanomaterials for Theranostics: Recent Advances and Future Challenges. Chem. Rev. 115, 327-394, (2015).

[5] Rahman, M., Laurent, S., Tawil, N., Yahia, L. H. \& Mahmoudi, M. Protein-Nanoparticle Interactions: The Bio-Nano Interface. (Springer Berlin Heidelberg, 2013).

[6] The two directions of cancer nanomedicine. Nat. Nanotechnol. 14, 1083-1083, (2019).

[7] Wilhelm, S. et al. Analysis of nanoparticle delivery to tumours. Nat. Rev. Mater. 1, (2016).

[8] Dai, Q. et al. Quantifying the Ligand-Coated Nanoparticle Delivery to Cancer Cells in Solid Tumors. ACS Nano 12, 8423-8435, (2018).

[9] Walkey, C. D. \& Chan, W. C. W. Understanding and controlling the interaction of nanomaterials with proteins in a physiological environment. Chem. Soc. Rev. 41, 2780-2799, (2012).

[10] Monopoli, M. P., Bombelli, F. B. \& Dawson, K. A. Nanobiotechnology: Nanoparticle coronas take shape. Nat. Nanotechnol. 6, 11-12, (2011).

[11] Cedervall, T. et al. Understanding the nanoparticle-protein corona using methods to quantify exchange rates and affinities of proteins for nanoparticles. Proc. Natl. Acad. Sci. U.S.A. 104, 2050, (2007).

[12] Lima, R., Seabra Amedea, B. \& Durán, N. Silver nanoparticles: a brief review of cytotoxicity and genotoxicity of chemically and biogenically synthesized nanoparticles. J. Appl. Toxicol. 32, 867-879, (2012).

[13] Skalska, J. \& Strużyńska, L. Toxic effects of silver nanoparticles in mammals - does a risk of neurotoxicity exist? Folia Neuropathol. 53, 281-300, (2015).

[14] Foldbjerg, R. B. \& Autrup, H. Mechanisms of Silver Nanoparticle Toxicity. Arch. Bas. App. Med. 1, 5-15, (2013).

[15] Samberg, M. E. \& Monteiro-Riviere, N. A. in Encyclopedia of Nanotechnology (ed Bharat Bhushan) 1069-1077 (Springer Netherlands, 2012).

[16] Durán, N., Nakazato, G. \& Seabra, A. B. Antimicrobial activity of biogenic silver nanoparticles, and silver chloride nanoparticles: an overview and comments. Appl. Microbiol. Biotechnol. 100, 6555-6570, (2016). 
[17] Aggarwal, P., Hall, J. B., McLeland, C. B., Dobrovolskaia, M. A. \& McNeil, S. E. Nanoparticle interaction with plasma proteins as it relates to particle biodistribution, biocompatibility and therapeutic efficacy. Adv Drug Deliv Rev 61, 428-437, (2009).

[18] Liu, N., Tang, M. \& Ding, J. The interaction between nanoparticles-protein corona complex and cells and its toxic effect on cells. Chemosphere 245, 125624, (2020).

[19] Barbalinardo, M., Caicci, F., Cavallini, M. \& Gentili, D. Protein Corona Mediated Uptake and Cytotoxicity of Silver Nanoparticles in Mouse Embryonic Fibroblast. Small 14, 1801219, (2018).

[20] Decataldo, F. et al. Organic Electrochemical Transistors for Real-Time Monitoring of In Vitro Silver Nanoparticle Toxicity. Adv. Biosys. 4, 1900204, (2020).

[21] Harder, P., Grunze, M., Dahint, R., Whitesides, G. M. \& Laibinis, P. E. Molecular Conformation in Oligo(ethylene glycol)-Terminated Self-Assembled Monolayers on Gold and Silver Surfaces Determines Their Ability To Resist Protein Adsorption. J. Phys. Chem. B 102, 426-436, (1998).

[22] Herrwerth, S., Eck, W., Reinhardt, S. \& Grunze, M. Factors that determine the protein resistance of oligoether self-assembled monolayers - Internal hydrophilicity, terminal hydrophilicity, and lateral packing density. J. Am. Chem. Soc. 125, 9359-9366, (2003).

[23] Li, L. Y., Chen, S. F., Zheng, J., Ratner, B. D. \& Jiang, S. Y. Protein adsorption on oligo(ethylene glycol)-terminated alkanethiolate self-assembled monolayers: The molecular basis for nonfouling behavior. J. Phys. Chem. B 109, 2934-2941, (2005).

[24] Gentili, D. et al. Integration of organic electrochemical transistors and immuno-affinity membranes for label-free detection of interleukin-6 in the physiological concentration range through antibody-antigen recognition. J. Mater. Chem. B 6, 5400-5406, (2018).

[25] Prime, K. L. \& Whitesides, G. M. Self-assembled organic monolayers: model systems for studying adsorption of proteins at surfaces. Science 252, 1164-1167, (1991).

[26] Prime, K. L. \& Whitesides, G. M. Adsorption of proteins onto surfaces containing endattached oligo(ethylene oxide): a model system using self-assembled monolayers. J. Am. Chem. Soc. 115, 10714-10721, (1993).

[27] Duchesne, L., Gentili, D., Comes-Franchini, M. \& Fernig, D. G. Robust Ligand Shells for Biological Applications of Gold Nanoparticles. Langmuir 24, 13572-13580, (2008).

[28] Bastus, N. G., Merkoci, F., Piella, J. \& Puntes, V. Synthesis of Highly Monodisperse CitrateStabilized Silver Nanoparticles of up to $200 \mathrm{~nm}$ : Kinetic Control and Catalytic Properties. Chem. Mater. 26, 2836-2846, (2014).

[29] Love, J. C., Estroff, L. A., Kriebel, J. K., Nuzzo, R. G. \& Whitesides, G. M. Self-Assembled Monolayers of Thiolates on Metals as a Form of Nanotechnology. Chem. Rev. 105, 11031170, (2005).

[30] Hinterwirth, H. et al. Quantifying Thiol Ligand Density of Self-Assembled Monolayers on Gold Nanoparticles by Inductively Coupled Plasma-Mass Spectrometry. ACS Nano 7, 11291136, (2013).

[31] Torelli, M. D. et al. Quantitative Determination of Ligand Densities on Nanomaterials by Xray Photoelectron Spectroscopy. ACS Appl. Mater. Interfaces 7, 1720-1725, (2015).

[32] Gentili, D., Ori, G., Ortolani, L., Morandi, V. \& Cavallini, M. Cooperative and Reversible Anisotropic Assembly of Gold Nanoparticles by Modulation of Noncovalent Interparticle Interactions. ChemNanoMat 3, 874-878, (2017). 
[33] Chen, H., Kou, X., Yang, Z., Ni, W. \& Wang, J. Shape- and size-dependent refractive index sensitivity of gold nanoparticles. Langmuir 24, 5233-5237, (2008).

[34] Ishihama, Y. et al. Exponentially modified protein abundance index (emPAI) for estimation of absolute protein amount in proteomics by the number of sequenced peptides per protein. Mol. Cell. Proteomics 4, 1265-1272, (2005).

[35] Unsworth, L. D., Sheardown, H. \& Brash, J. L. Protein-Resistant Poly(ethylene oxide)Grafted Surfaces: Chain Density-Dependent Multiple Mechanisms of Action. Langmuir 24, 1924-1929, (2008).

[36] Walkey, C. D., Olsen, J. B., Guo, H., Emili, A. \& Chan, W. C. Nanoparticle size and surface chemistry determine serum protein adsorption and macrophage uptake. J. Am. Chem. Soc. 134, 2139-2147, (2012).

[37] Wagner, S. et al. Uptake mechanism of ApoE-modified nanoparticles on brain capillary endothelial cells as a blood-brain barrier model. PLoS One 7, e32568, (2012).

[38] Barran-Berdon, A. L. et al. Time evolution of nanoparticle-protein corona in human plasma: relevance for targeted drug delivery. Langmuir 29, 6485-6494, (2013).

[39] Gao, H. \& He, Q. The interaction of nanoparticles with plasma proteins and the consequent influence on nanoparticles behavior. Expert Opin. Drug Deliv. 11, 409-420, (2014).

[40] Nguyen, V. H. et al. Modulation of serum albumin protein corona for exploring cellular behaviors of fattigation-platform nanoparticles. Colloids Surf B Biointerfaces 170, 179-186, (2018).

[41] Yan, Y. et al. Differential roles of the protein corona in the cellular uptake of nanoporous polymer particles by monocyte and macrophage cell lines. ACS Nano 7, 10960-10970, (2013).

[42] Akinc, A. et al. Targeted delivery of RNAi therapeutics with endogenous and exogenous ligand-based mechanisms. Mol. Ther. 18, 1357-1364, (2010).

[43] Liu, K., Liu, P.-c., Liu, R. \& Wu, X. Dual AO/EB staining to detect apoptosis in osteosarcoma cells compared with flow cytometry. Med. Sci. Monit. Basic Res. 21, 15-20, (2015).

[44] AshaRani, P. V., Low Kah Mun, G., Hande, M. P. \& Valiyaveettil, S. Cytotoxicity and Genotoxicity of Silver Nanoparticles in Human Cells. ACS Nano 3, 279-290, (2009).

[45] Miura, N. \& Shinohara, Y. Cytotoxic effect and apoptosis induction by silver nanoparticles in HeLa cells. Biochem. Biophys. Res. Commun. 390, 733-737, (2009).

[46] Lee, Y.-H. et al. Cytotoxicity, oxidative stress, apoptosis and the autophagic effects of silver nanoparticles in mouse embryonic fibroblasts. Biomaterials 35, 4706-4715, (2014).

[47] Hsin, Y.-H. et al. The apoptotic effect of nanosilver is mediated by a ROS- and JNKdependent mechanism involving the mitochondrial pathway in NIH3T3 cells. Toxicol. Lett. 179, 130-139, (2008).

[48] Caballero-Díaz, E. et al. The Toxicity of Silver Nanoparticles Depends on Their Uptake by Cells and Thus on Their Surface Chemistry. Part. Part. Syst. Charact. 30, 1079-1085, (2013).

[49] Hühn, J. et al. Selected Standard Protocols for the Synthesis, Phase Transfer, and Characterization of Inorganic Colloidal Nanoparticles. Chem. Mater. 29, 399-461, (2016).

[50] Riener, C. K., Kada, G. \& Gruber, H. J. Quick measurement of protein sulfhydryls with Ellman's reagent and with 4,4'-dithiodipyridine. Anal. Bioanal. Chem. 373, 266-276, (2002).

[51] Shevchenko, A., Tomas, H., Havli, J., Olsen, J. V. \& Mann, M. In-gel digestion for mass spectrometric characterization of proteins and proteomes. Nat. Protoc. 1, 2856, (2007).

[52] Barbalinardo, M. et al. Data-Matrix Technology for Multiparameter Monitoring of Cell Cultures. Small Methods 2, 1700377, (2018). 
[53] Twentyman, P. R. \& Luscombe, M. A study of some variables in a tetrazolium dye (MTT) based assay for cell growth and chemosensitivity. Br. J. Cancer 56, 279-285, (1987).

[54] Squier, M. K. \& Cohen, J. J. Standard quantitative assays for apoptosis. Mol. Biotechnol. 19, 305-312, (2001). 